Revista de MATEMÁticA: TeORÍA y APliCACIONEs 2015 22(2) : 275-291

CIMPA - UCR ISSN: 1409-2433 (PRINT), 2215-3373 (ONLINE)

\title{
LA ESPECIE DE LOS PULPOS
}

\section{THE OCTOPUSES SPECIES}

\author{
EDDY PARIGUAN* JUAN SEBASTIÁN RODRÍGUEZ ${ }^{\dagger}$
}

Received: 24/Jul/2013; Revised: 20/Mar/2015;

Accepted: 7/May/2015

*Departamento de Matemáticas, Pontificia Universidad Javeriana. Bogotá, Colombia. E-Mail: epariguan@javeriana.edu.co

${ }^{\dagger}$ Instituto de Matemáticas Puras y Aplicadas, IMPA, Rio de Janeiro, Brasil. E-Mail: juanr@impa.br 


\begin{abstract}
Resumen
El objetivo principal de este trabajo es introducir la especie de los pulpos en combinatoria enumerativa. Demostraremos además la validez de ciertas ecuaciones combinatorias sugeridas por Bergeron et al. en [3].
\end{abstract}

Palabras clave: combinatoria enumerativa; funciones generatrices; especies combinatorias.

\begin{abstract}
The main goal of this paper is introduce the species of octopuse in enumerative combinatoricss. We also prove the validity of some combinatorial equations suggested by Bergeron et al. in [3].
\end{abstract}

Keywords: enumerative combinatorics; generating functions; combinatorial species.

Mathematics Subject Classification: 05C30, 05A15, 05A99.

\title{
1 Introducción
}

En los útimos años se ha visto una unificación de las matemáticas a través de conceptos fundamentales de la teoría de categorías. Por ejemplo, los conceptos de categorías, functores y transformaciones naturales proporcionan una base matemática a la intuición combinatoria a través de la idea de especie combinatoria, o también conocida como especie de estructuras. Algunas teorías útiles que explican técnicas algebraicas como la teoría de especies combinatorias, la inversión de Möbius [10], la composición particional [4], la reducción de álgebras de incidencia [9], el cálculo umbral y las álgebras de Hopf [11], entre otras, surgen para esclarecer y fortalecer las bases de la combinatoria enumerativa.

La teoría de especies combinatorias fue introducida en el año 1981 por el matemático canadiense André Joyal, [6] y ha sido posteriormente desarrollada por F. Bergeron, G. Labelle y P. Leroux en [3]. Esta teoría sirve como herramienta para analizar estructuras discretas, tanto etiquetadas como no etiquetadas, utilizando diversos tipos de series. Algunos ejemplos de estructuras discretas son árboles, grafos, permutaciones, funciones, ciclos, grupos finitos, entre otras, [12]. El caso cuántico ha sido estudiado en [5].

En este trabajo presentaremos una breve revisión de las ideas generales de la teoría de especies, introduciremos la especie de los Pulpos y finalmente daremos pruebas combinatorias de algunas propiedades generales de la misma.

Para una compresión de las ideas desarrolladas en este trabajo asumimos que el lector está familiarizado con la teoría de especies combinatorias desarrollada en [3]. 


\section{Teoría de especies combinatorias}

En esta sección realizamos una breve revisión de los conceptos básicos de la teoría de especies. A lo largo de este trabajo utilizaremos la siguiente notación: para cada conjunto finito $U,|U|$ denota la cardinalidad de $U$ y para todo $n \in \mathbb{N}$, $[n]=\{1,2, \cdots, n\}$.

Definición 2.1. Una especie de estructura $F$ es una regla que asigna a cada conjunto finito $U$ un conjunto finito $F[U]$ y a cada biyección $\sigma: U \longrightarrow$ Ventre conjuntos finitos $U$ y $V$ una función $F[\sigma]: F[U] \longrightarrow F[V]$. La función $F[\sigma]$ satisface las siguientes propiedades functoriales

- Para cada par de biyecciones entre conjuntos finitos $\sigma: U \longrightarrow V y$ $\pi: V \longrightarrow W$, se satisface que $F[\pi \circ \sigma]=F[\pi] \circ F[\sigma]$.

- Si $\mathrm{id}_{\mathrm{U}}: \mathrm{U} \longrightarrow \mathrm{U}$ es la función identidad de $U$ entonces $F\left[\mathrm{id}_{\mathrm{U}}\right]=\mathrm{id}_{\mathrm{F}[\mathrm{U}]}$.

Un elemento $s \in F[U]$ se llama una $F$-estructura sobre $U$ o simplemente una especie de $F$ estructuras sobre $U$. La función $F[\sigma]$ se llama transporte de $F$-estructuras a lo largo de $\sigma$.

Observación 2.2. Una especie de estructuras $F$ es un functor $F: \mathbb{B} \longrightarrow$ Set entre la categoría $\mathbb{B}$ de conjuntos finitos y biyecciones y la categoría Set de conjuntos finitos y funciones. El lector interesado en teoría de categorías puede revisar [8].

Algunos ejemplos de especies son:

1. La especie de conjuntos $E$, definida mediante la expresión $E[U]=\{U\}$. Para cada conjunto finito $U$ existe una única $E$-estructura, el mismo conjunto $U$.

2. La especie singular $X$ definida mediante la expresión

$$
X[U]=\left\{\begin{array}{cl}
\{U\}, & \text { si }|U|=1 \\
\emptyset, & \text { en otro caso. }
\end{array}\right.
$$

3. La especie característica del conjunto vacío 1, definida mediante la expresión

$$
1[U]=\left\{\begin{array}{cl}
\{U\}, & \text { si } U=\emptyset \\
\emptyset, & \text { en otro caso }
\end{array}\right.
$$


Existen diversos mecanismos que permiten definir una especie de estructura, a través de axiomas teóricos de teoría de conjuntos, a través de operaciones entre especies de estructuras, a través de soluciones de ecuaciones funcionales, etc. En este trabajo solo haremos una breve descripción de la construcción de especies a través de operaciones. Puede revisar el resto de las construcciones en [3].

A cada especie de estructura $F$ se le puede asociar tres tipos distintos de series formales, las cuales están directamente relacionadas con el conteo de $F$ estructuras. Una $F$-estructura $s \in F[U]$ sobre el conjunto $U$ es una estructura etiquetada, mientras que una estructura no etiquetada es una clase de isomorfismo de $F$-estructuras.
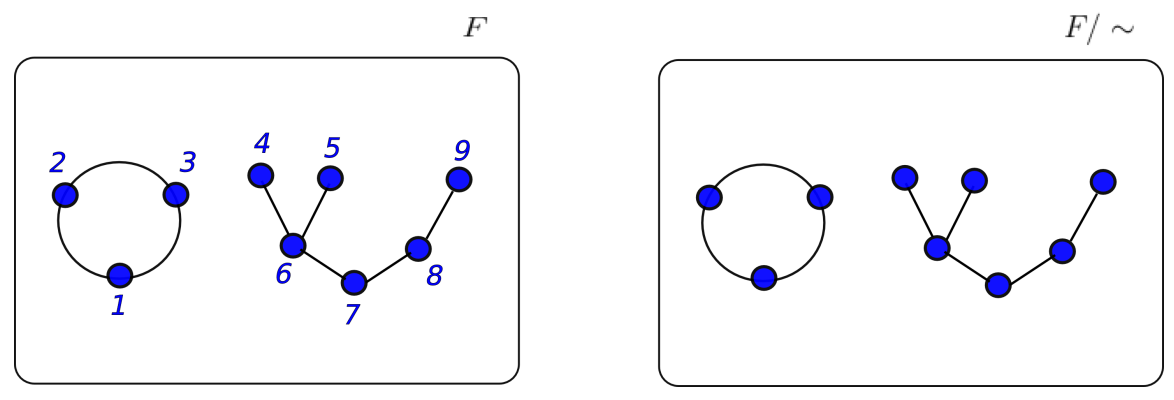

Figura 1: Estructura etiquetada y estructura no etiquetada.

Las series asociadas a una especie $F$ son las siguientes:

Definición 2.3 (Serie generatriz de tipo exponencial). Sea F una especie de estructura, denotemos por $f_{n}=|F[n]|$ el número de F-estructuras sobre un conjunto de tamaño $n$. La serie formal dada por la expresión

$$
F(x)=\sum_{n=0}^{\infty} f_{n} \frac{x^{n}}{n !}
$$

se llama serie generatriz de tipo exponencial asociada a la especie $F$.

Definición 2.4 (Serie generatriz). Sea F una especie de estructura, denotemos por $\widetilde{f_{n}}$ el número de F-estructuras no etiquetadas sobre un conjunto de tamaño n. La serie formal dada por la expresión

$$
\widetilde{F}(x)=\sum_{n=0}^{\infty} \widetilde{f_{n}} x^{n}
$$

se llama serie generatriz asociada a la especie $F$. 
Definición 2.5 (Serie indicadora de ciclos). Sea $F$ una especie de estructura, sea $U$ un conjunto finito y sea $\sigma$ una permutación de $U$. Denotaremos por $\left(\sigma_{1}, \sigma_{2}, \cdots\right)$ al tipo de ciclo de la permutación $\sigma$. La serie formal dada por la expresión

$$
Z_{F}\left(x_{1}, x_{2}, \cdots\right)=\sum_{n \geq 0} \frac{1}{n !}\left(\sum_{\sigma \in S_{n}} \operatorname{fix} F[\sigma] x_{1}^{\sigma_{1}} x_{2}^{\sigma_{2}} \cdots\right)
$$

donde $S_{n}$ representa el grupo simétrico en n-letras y fix $F[\sigma]$ el número de $F$ estructuras sobre $[n]$ que están fijas por $F[\sigma]$, se llama serie indicadora de ciclos asociada a la especie $F$.

Las series asociadas a una especie están directamente relacionadas con el conteo de $F$-estructuras etiquetadas y no etiquetadas (clases de isomorfismos). El cálculo de estas series es complicado. En algunos casos, se requiere el uso de operaciones combinatorias entre especies de estructuras y muchas veces, para el caso de la serie generatriz es necesario usar la serie indicadora de ciclos. La serie indicadora de ciclos está relacionada con la teoría de Polya, [13].

Ejemplo 2.6. Consideremos las especies $L, X, S, E$ y $C$ de ordenes lineales, singular, permutaciones, conjuntos y permutaciones cíclicas respectivamente. Las series asociadas a estas especies están dadas por

1. Serie generatriz de tipo exponencial

- $L(x)=\frac{1}{1-x}, \quad X(x)=x$.

- $S(x)=\frac{1}{1-x}, \quad E(x)=e^{x}$

- $C(x)=-\log (1-x)$.

2. Serie generatriz

- $\widetilde{L}(x)=\frac{1}{1-x}, \quad \widetilde{X}(x)=x$.

- $\widetilde{S}(x)=\prod_{k=1}^{\infty} \frac{1}{1-x^{k}}, \widetilde{E}(x)=\frac{1}{1-x}$.

- $\widetilde{C}(x)=\frac{x}{1-x}$.

3. Serie indicadora de ciclos

- $Z_{L}\left(x_{1}, x_{2}, \cdots\right)=\frac{1}{1-x_{1}}, \quad Z_{X}\left(x_{1}, x_{2}, \cdots\right)=x_{1}$. 
- $Z_{S}\left(x_{1}, x_{2}, \cdots\right)=\frac{1}{\left(1-x_{1}\right)\left(1-x_{2}\right) \cdots}$.

- $Z_{E}\left(x_{1}, x_{2}, \cdots\right)=\exp \left(x_{1}+\frac{x_{2}}{2}+\frac{x_{3}}{3}+\cdots\right)$.

- $Z_{C}\left(x_{1}, x_{2}, \cdots\right)=\sum_{k=1}^{\infty} \frac{\phi(k)}{k !} \log \frac{1}{1-x_{k}}$, donde $\phi$ denota al función $\phi$ de Euler, definida como

$$
\phi(n)=|\{m \in \mathbb{N}: m<n y(n, m)=1\}| .
$$

Para finalizar esta sección vamos a recordar el concepto de especies isomorfas, el mismo será necesario en las próximas secciones.

Definición 2.7. Sean $F$ y $G$ dos especies de estructuras. Un isomorfismo de $F$ a $G$ es una familia de biyecciones $\alpha_{U}: F[U] \longrightarrow G[U]$ que satisface la siguiente condición natural, para cada biyección $\sigma: U \longrightarrow V$ entre conjuntos finitos, el siguiente diagrama es conmutativo

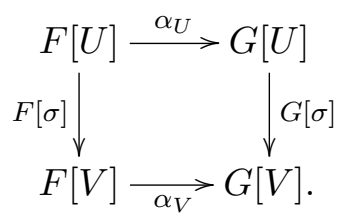

\section{Operaciones con especies}

A continuación vamos a presentar una pequeña revisión de las operaciones básicas entre especies para luego presentar la especie de los pulpos el cual es el objetivo principal de nuestro trabajo. Se requiere obtener especies de estructuras a través de "combinaciones" entre especies ya conocidas. Para definir una nueva especie solo debemos decir cómo se comporta la nueva regla sobre un conjunto finito y cómo se realiza el transporte de estructuras.

Definición 3.1 (Suma de especies). Sean F y $G$ dos especies de estructuras y sea $U$ un conjunto finito. La especie $F+G$, llamada la suma de las especies $F$ y G está dada por

1. $(F+G)[U]=F[U]+G[U]$, donde el símbolo + se entiende como unión disjunta. 
2. El transporte de estructuras a lo largo de la biyección $\sigma: U \longrightarrow V$, para cada $F+G$ estructura s, está dado por

$$
(F+G)[\sigma](s)= \begin{cases}F[\sigma](s), & \text { si } s \in F[U] \\ G[\sigma](s), & \text { si } s \in G[U]\end{cases}
$$
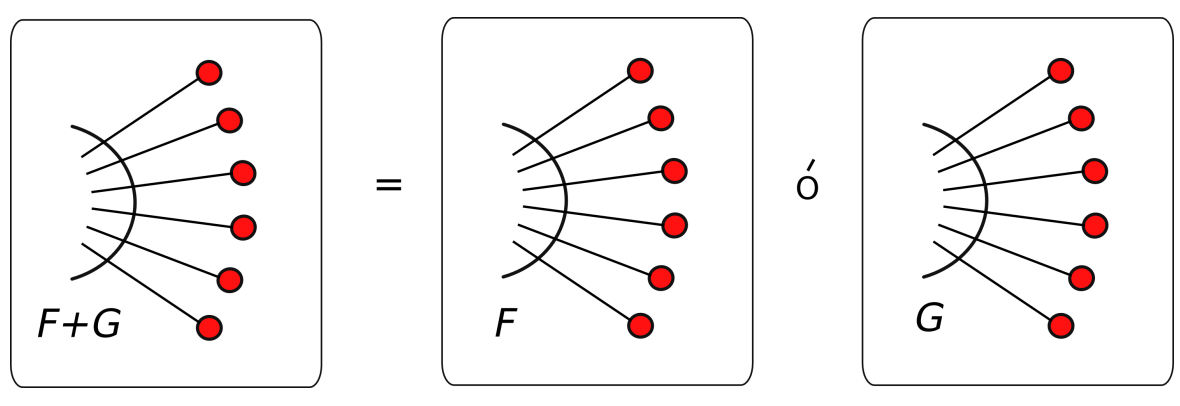

Figura 2: Representación gráfica de la suma de especies.

Definición 3.2 (Producto de especies). Sean F y G dos especies de estructuras y sea $U$ un conjunto finito. La especie FG llamada el producto de las especies F y G está dada por

1. Un par $s=(f, g)$, donde $f$ es una $F$-estructura sobre $U_{1} \subset U$; g es una $G$-estructura sobre $U_{2} \subset U$ y $\left(U_{1}, U_{2}\right)$ es una descomposición de $U$ en suma directa. Explicítamente

$$
(F G)[U]:=\sum_{\left(U_{1}, U_{2}\right)} F\left[U_{1}\right] \times G\left[U_{2}\right],
$$

donde la unión disjunta se toma sobre todas las posibles descomposiciones $\left(U_{1}, U_{2}\right)$ de $U$ en suma directa.

2. El transporte de estructuras a lo largo de la biyección $\sigma: U \longrightarrow V$, para cada FG estructura $s=(f, g)$, está dado por

$$
(F G)[\sigma](s)=\left(F\left[\sigma_{1}\right](f), G\left[\sigma_{2}\right](g)\right),
$$

donde $\sigma_{i}$ representa la restricción de $\sigma$ a $U_{i}, i=1,2$. 


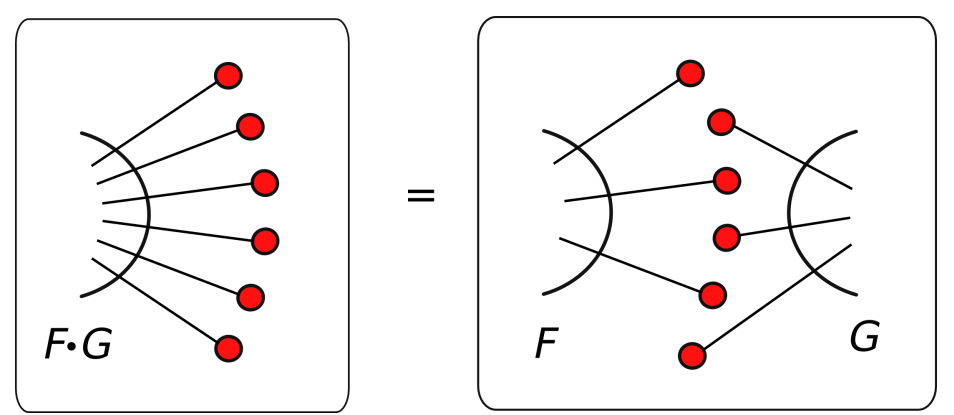

Figura 3: Representación gráfica del producto de especies.

Definición 3.3 (Sustitución de especies). Sean F y $G$ dos especies de estructuras tales que $G[\emptyset]=\emptyset$. La especie $F \circ G$ llamada composición particional de $G$ en F está dada por

1. Una tupla $s=(\pi, \varphi, \gamma)$ donde, $\pi$ es una partición de $U ; \varphi$ es una $F$ estructura sobre el conjunto de clases de $\pi$ y $\gamma=\left(\gamma_{p}\right)_{p \in \pi}$ donde para cada clase $p \in \pi$, $\gamma_{p}$ es una $G$-estructura sobre $p$. Explicítamente

$$
(F \circ G)[U]:=\sum_{\pi \in \operatorname{Par}(U)} F[\pi] \times \prod_{p \in \pi} G[p],
$$

donde la unión disjunta se toma sobre todas las posibles particiones $\pi$ de $U$, es decir, $\pi \in \operatorname{Par}(U)$.

2. El transporte de estructuras a lo largo de la biyección $\sigma: U \longrightarrow V$ para cada $F \circ G$ estructura $s=\left(\pi, \varphi,\left(\gamma_{p}\right)_{p \in \pi}\right)$, está dado por

$$
(F \circ G)[\sigma](s)=\left(\bar{\pi}, \bar{\varphi},\left(\bar{\gamma}_{\bar{p}}\right)_{\bar{p} \in \bar{\pi}}\right),
$$

donde

- $\bar{\pi}$ es la partición de $V$ obtenida a través del transporte de $\pi$ a lo largo de $\sigma$,

- para cada $\bar{p} \in \sigma(p) \in \bar{\pi}$, la estructura $\bar{\gamma}_{\bar{p}}$ se obtiene de la estructura $\gamma_{p}$ a través del $G$-transporte a lo largo de $\left.\sigma\right|_{p}$,

- la estructura $\bar{\varphi}$ se obtiene de la estructura $\varphi$ a través del F-transporte a lo largo de la biyección $\bar{\sigma}$ inducida sobre $\pi$ por $\sigma$. 


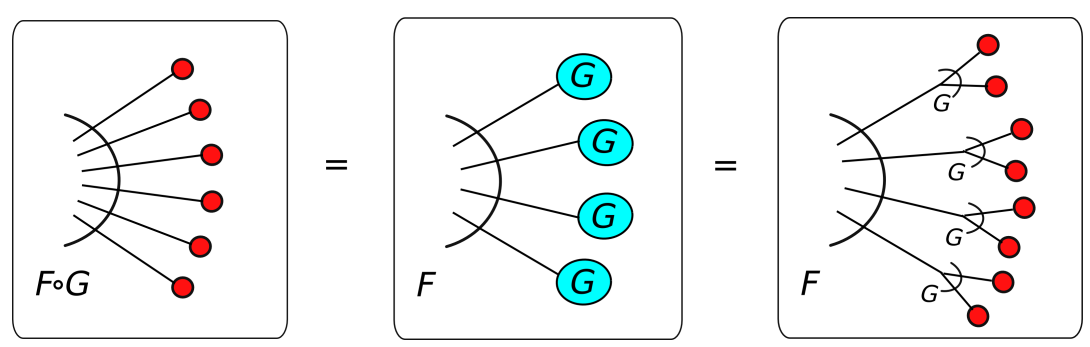

Figura 4: Representación gráfica de la sustitución de especies.

Definición 3.4 (Derivada de una especie). Sea F una especie de estructuras. La especie $F^{\prime}$, llamada la derivada de la especie $F$, está dada por

1. $F^{\prime}[U]=F\left[U^{+}\right]$, donde $U^{+}=U \cup\{*\} y *$ es un elemento fuera del conjunto $U$.

2. El transporte de estructuras a lo largo de la biyección $\sigma: U \longrightarrow V$ para cada $F^{\prime}$ estructura s, está dado por

$$
F^{\prime}[\sigma](s)=F\left[\sigma^{+}\right](s),
$$

donde $\sigma^{+}: U \cup\{*\} \longrightarrow V \cup\{*\}$ es la extensión canónica de $\sigma$ que se obtiene como $\sigma^{+}(u)=\sigma(u)$ si $u \in U$ y $\sigma^{+}(*)=*$.

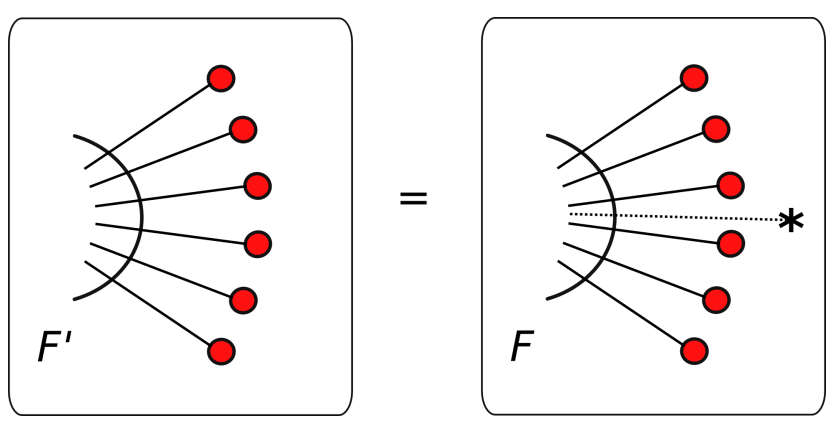

Figura 5: Representación gráfica de la derivada de especies.

Las operaciones combinatorias entre especies de estructuras producen nuevas estructuras, generalmente más complejas. Estas operaciones entre especies se corresponden con las operaciones entre series formales [7]. 


\section{La especie de los pulpos}

La especie de los pulpos es introducida por Bergeron en [2] como un modelo combinatorio para estudiar diversas familias de polinomios ortogonales, las cuales envuelven distintas expresiones que se demuestran combinatoriamente. Presentaremos una descripción combinatoria de la especie de los pulpos y demostraremos algunas identidades combinatorias que satisface esta especie.

Denotamos por Oct la especie de los pulpos. Gráficamente una típica estructura de tipo Oct sobre un conjunto finito $U$ es de la forma

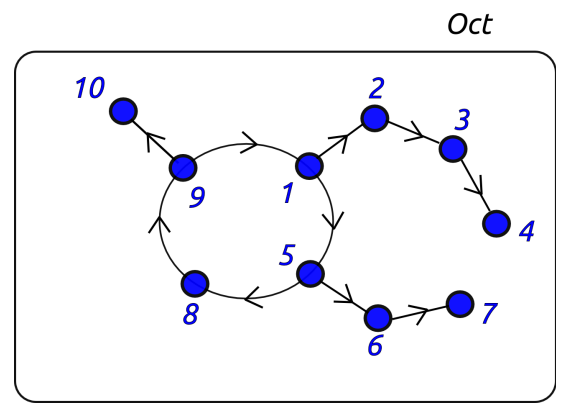

Figura 6: Oct-estructura sobre [10].

Observe que la especie Oct satisface la ecuación combinatoria Oct $=C$ o $L_{+}=C\left(L_{+}\right)$, donde $C$ representa la especie de permutaciones cíclicas y $L_{+}$ la especie de los ordenes lineales sin estructura en el vacío. Una Oct-estructura sobre $U$ consiste en una partición $\pi$ de $U$, un orden lineal sobre cada bloque de la partición $\pi$ y una permutación cíclica sobre $\pi$. Este hecho se ilustra en la Figura 7.

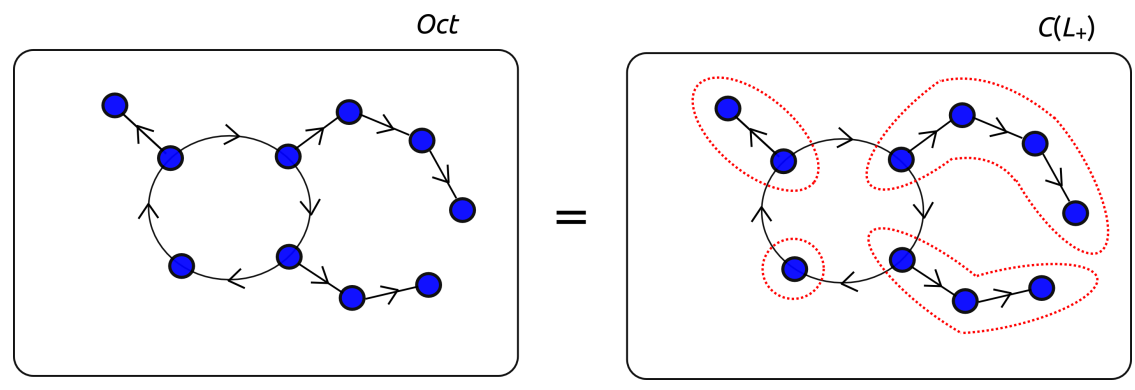

Figura 7: Composición de $C$ y $L_{+}$. 
Haciendo uso de la identidad combinatoria Oct $=C \circ L_{+}$, en términos de series generatrices de tipo exponencial se tiene que $\operatorname{Oct}(x)=\left(C \circ L_{+}\right)(x)$, de donde se sigue que

$$
\begin{aligned}
\operatorname{Oct}(x) & =C\left(L_{+}(x)\right) \\
& =C\left(\frac{x}{1-x}\right) \\
& =-\log \left(1-\frac{x}{1-x}\right) \\
& =-\log \left(\frac{1-2 x}{1-x}\right) \\
& =-\log (1-2 x)+\log (1-x) \\
& =C(2 x)-C(x) .
\end{aligned}
$$

Más aún se cumple la siguiente proposición:

Proposición 4.1. Considere Oct la especie de los pulpos, $C$ la especie de permutaciones cíclicas y $X$ la especie singular, entonces se satisface la siguiente ecuación combinatoria

$$
\text { Oct }+C=C(2 X)
$$

Prueba. Observe que una estructura de tipo $C(2 X)=C(X+X)$ sobre un conjunto finito $U$ consiste en los siguientes datos:

- Una partición $\pi$ del conjunto $U$.

- Para cada bloque $p$ de la partición $\pi$, una estructura de tipo $X+X$ sobre $p$.

- Un ciclo sobre $\pi$.

Note que cada $p \in \pi$ debe satisfacer $|p|=1$ y que una estructura de tipo $X+X$ sobre un conjunto unitario $\{u\}$ es un par $(u, i)$ con $i=1$ ó $i=2$. El elemento de la forma $(u, 1)$ lo vamos a pensar como el elemento $u$ cuadrado coloreado de color rojo y el elemento de la forma $(u, 2)$ como el elemento $u$ redondo coloreado de color verde. Es decir, una $C(2 X)$ estructura sobre el conjunto $U$ es un ciclo sobre $U$ donde cada elemento $u \in U$ puede estar coloreado de color rojo o de color verde, como se muestra en la Figura 8. 


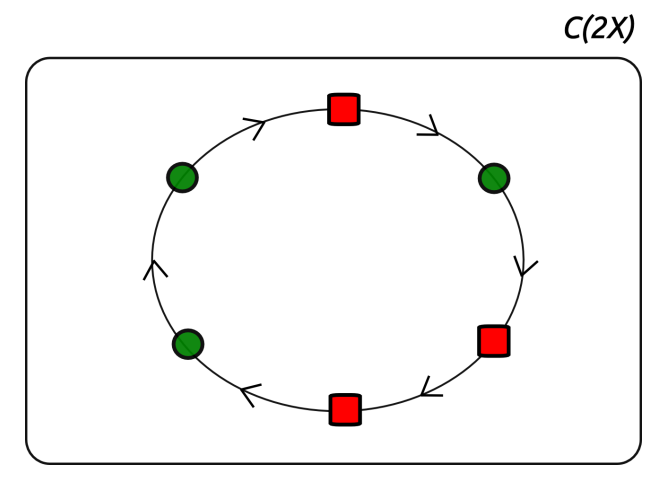

Figura 8: Ejemplo de una $C(2 X)$-estructura.

Queremos probar entonces que las especies Oct $+C$ y $C(2 X)$ son isomorfas. Para cada conjunto finito $U$ definamos $\alpha_{U}:($ Oct $+C)[U] \longrightarrow C(2 X)[U]$ gráficamente por:
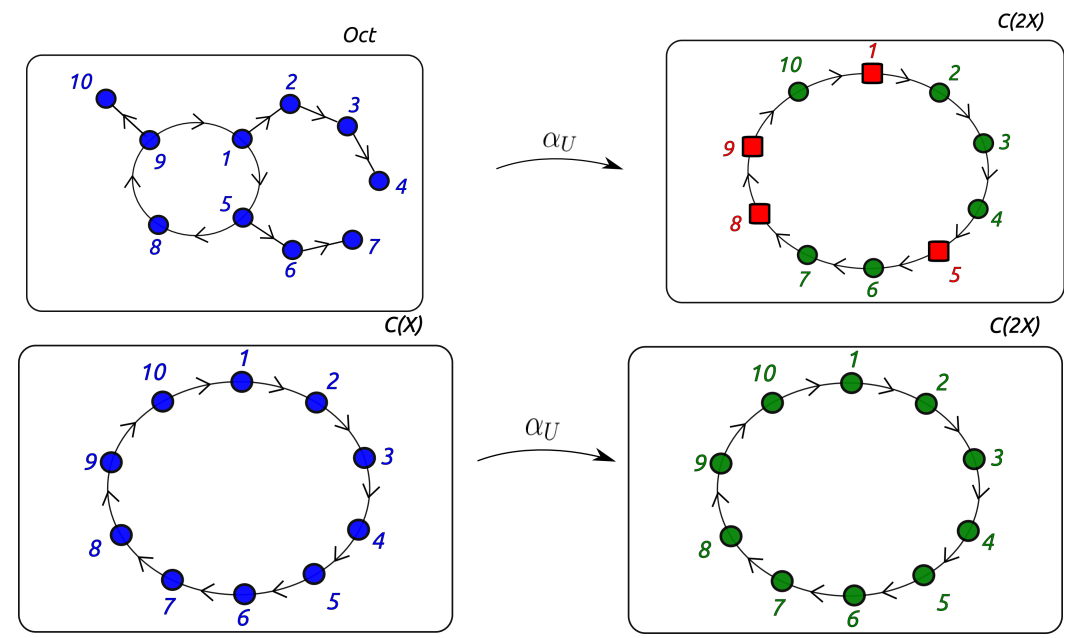

Claramente $\alpha_{U}$ tiene inversa y por lo tanto es una biyección. Más aún, para cada biyección $\sigma: U \longrightarrow V$ entre conjuntos finitos el siguiente diagrama es conmutativo

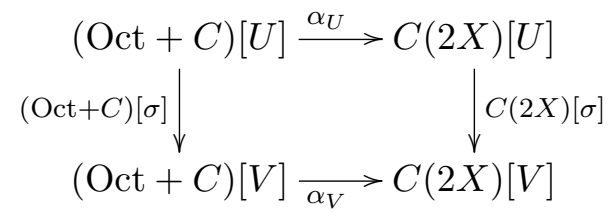


En efecto, sea $s \in($ Oct $+C)[U]$. Como la conmutatividad del diagrama es inmediata para elementos de $C[U]$, podemos suponer que $s \in \operatorname{Oct}[U]$. Es claro que dos $C(2 X)$-estructuras $t, t^{\prime}$ sobre $V$ son iguales si y sólo si cualquier $v \in V$ tiene el mismo color en $t$ y $t^{\prime}$, y además si el predecesor y sucesor de $v$ son iguales en $t$ y $t^{\prime}$.

Observe que si $v \in V$ es cuadrado de color rojo en $\left(\alpha_{V} \circ(\right.$ Oct $\left.+C)[\sigma]\right)(s)$, entonces $v$ es un elemento mínimo de algún tentáculo de $(\mathrm{Oct}+C)[\sigma](s)$ y como el transporte de estructuras en Oct consiste en reetiquetar de acuerdo a $\sigma$, entonces $\sigma^{-1}(v)$ es un elemento mínimo de algún tentáculo de $s$. Luego, $\sigma^{-1}(v)$ es cuadrado de color rojo en $\alpha_{U}(s)$ y por lo tanto $v$ es cuadrado de color rojo en $\left(C(2 X)[\sigma] \circ \alpha_{U}\right)(s)$. Análogamente se tiene que si $v$ es redondo de color verde en $\left(\alpha_{V} \circ(\right.$ Oct $\left.+C)[\sigma]\right)(s)$, entonces $v$ es redondo de color verde en $\left(C(2 X)[\sigma] \circ \alpha_{U}\right)(s)$. Por lo tanto cualquier $v \in V$ tiene el mismo color en $\left(\alpha_{V} \circ(\right.$ Oct $\left.+C)[\sigma]\right)(s)$ y $\left(C(2 X)[\sigma] \circ \alpha_{U}\right)(s)$.

Sean $v^{\prime}$ y $v^{\prime \prime}$ el predecesor y sucesor de $v$ en $\left(C(2 X)[\sigma] \circ \alpha_{U}\right)(s)$, respectivamente. Luego, $\sigma^{-1}\left(v^{\prime}\right)$ y $\sigma^{-1}\left(v^{\prime \prime}\right)$ son el predecesor y sucesor de $\sigma^{-1}(v)$ en $\alpha_{U}(s)$, respectivamente. De donde se sigue facilmente que $v^{\prime}$ y $v^{\prime \prime}$ son el predecesor y sucesor de $v$ en $\left(\alpha_{V} \circ(\right.$ Oct $\left.+C)[\sigma]\right)(s)$, respectivamente. Es decir, el predecesor y sucesor de $v$ son iguales en $\left(\alpha_{V} \circ(\right.$ Oct $\left.+C)[\sigma]\right)(s)$ y $\left(C(2 X)[\sigma] \circ \alpha_{U}\right)(s)$. Por lo tanto $\left(\alpha_{V} \circ(\right.$ Oct $\left.+C)[\sigma]\right)=\left(C(2 X)[\sigma] \circ \alpha_{U}\right)$. Es decir $\alpha$ es un isomorfismo de especies y se satisface la ecuación

$$
\text { Oct }+C=C(2 X) \text {. }
$$

Por ejemplo si $\sigma:[10] \longrightarrow[10]$ es la permutación dada por la matriz

$$
\sigma=\left(\begin{array}{cccccccccc}
1 & 2 & 3 & 4 & 5 & 6 & 7 & 8 & 9 & 10 \\
5 & 3 & 10 & 4 & 2 & 1 & 8 & 6 & 7 & 9
\end{array}\right),
$$

entonces el diagrama para un elemento de Oct[10] es el mostrado en la Figura 9.

Lo mismo para un elemento de $C[10]$, es decir $\alpha$ es un isomorfismo de especies y por lo tanto Oct $+C=C(2 X)$.

\section{Series asociadas a la especie de los pulpos}

Sean $F, G$ y $H$ tres especies de estructuras tales que $F=G \circ H$, entonces se satisfacen las siguientes identidades:

1. $F(x)=G(H(x))$.

2. $\widetilde{F}(x)=Z_{G}\left(\widetilde{H}(x), \widetilde{H}\left(x^{2}\right), \widetilde{H}\left(x^{3}\right), \cdots\right)$ 


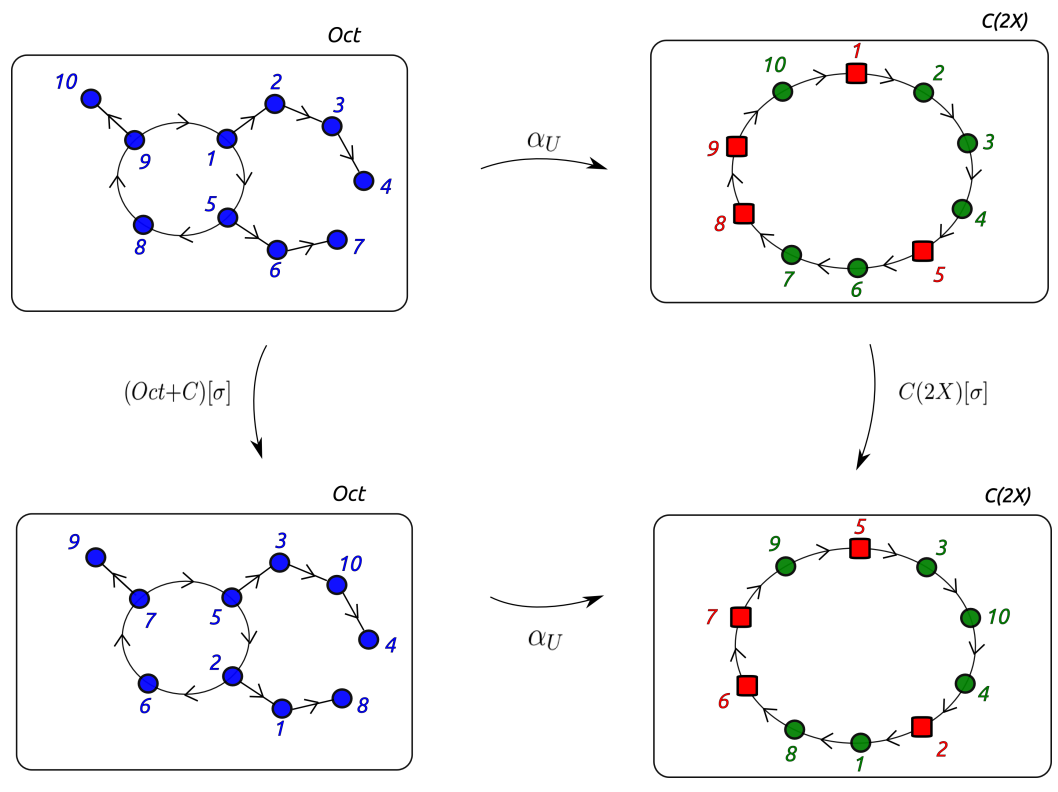

Figura 9: Diagrama para un elemento de Oct[10].

3. $Z_{F}\left(x_{1}, x_{2}, x_{3}, \cdots\right)=Z_{G}\left(Z_{H}\left(x_{1}, x_{2}, x_{3}, \cdots\right), Z_{H}\left(x_{2}, x_{4}, x_{6}, \cdots\right), \cdots\right)$.

Sean $S, E$ y $C$ las especies de permutaciones, conjuntos y permutaciones cíclicas, respectivamente. Luego es bien conocida la siguiente identidad combinatoria $S=E \circ C$, [3], entonces tenemos lo siguiente:

- $\frac{1}{1-x}=\exp C(x)$.

- $\frac{1}{1-x_{1}} \frac{1}{1-x_{2}} \frac{1}{1-x_{3}} \cdots=\exp \sum_{k \geq 1} \frac{1}{k} Z_{C}\left(x_{k}, x_{2 k}, x_{3 k}, \cdots\right)$.

Observe que $S=E \circ C$ implica $S(x)=E(C(x))$ y, por lo tanto

$$
S(x)=\frac{1}{1-x}=E(C(x))=\exp C(x) .
$$

Análogamente obtenemos la segunda identidad. Usando estas igualdades podemos probar el Lema 5.1.

Lema 5.1. Sea $C$ la especie de permutaciones cíclicas, las siguientes identidades se satisfacen 
- $C(x)=-\log (1-x)$.

- $Z_{C}\left(x_{1}, x_{2}, \cdots\right)=\sum_{k \geq 1} \frac{\phi(k)}{k} \log \left(\frac{1}{1-x_{k}}\right)$, donde $\phi$ representa la función de Euler, [1].

Proposición 5.2. Sea Oct la especie de pulpos, las series asociadas a esta especie satisfacen las siguientes identidades

1. $\operatorname{Oct}(x)=\sum_{k \geq 1} \frac{2^{k}-1}{k !} x^{k}$.

2. $Z_{\text {Oct }}\left(x_{1}, x_{2}, x_{2}, \cdots\right)=\sum_{k \geq 1} \frac{\phi(k)}{k} \log \left(\frac{1-x_{k}}{1-2 x_{k}}\right)$.

Prueba.

1. Gracias a la Proposición 4.1 tenemos que

$$
\operatorname{Oct}(x)=C(2 x)-C(x),
$$

luego

$$
\begin{aligned}
\operatorname{Oct}(x) & =\sum_{k \geq 1} \frac{1}{k}(2 x)^{k}-\sum_{k \geq 1} \frac{1}{k}(x)^{k} \\
& =\sum_{k \geq 1} \frac{2^{k}-1}{k} x^{k} .
\end{aligned}
$$

2. Como Oct $=C\left(L_{+}\right)$, entonces utilizando el Lema 5.1 y la identidad

$$
Z_{L_{+}}\left(x_{1}, x_{2}, x_{3}, \cdots\right)=\frac{2-x_{1}}{1-x_{1}}
$$

se tiene que

$$
\begin{aligned}
Z_{\mathrm{Oct}}\left(x_{1}, x_{2}, x_{3}, \cdots\right) & =Z_{C}\left(\frac{2-x_{1}}{1-x_{1}}, \frac{2-x_{2}}{1-x_{2}}, \cdots\right) \\
& =\sum_{k \geq 1} \frac{\phi(k)}{k} \log \left(\frac{1-x_{k}}{1-2 x_{k}}\right) .
\end{aligned}
$$

Corolario 5.3. El número de pulpos etiquetados sobre un conjunto $U$ de tamaño $k$ está dado por

$$
|\operatorname{Oct}[k]|=(k-1) !\left(2^{k}-1\right) \text {. }
$$




\section{Agradecimientos}

A Paola Vargas por su excelente trabajo desarrollado en el curso de Teoría de Especies Combinatorias. El primer autor agradece a la Pontificia Univeridad Javeriana por su apoyo parcial para la realización de este trabajo, el cual se encuentra enmarcado dentro del proyecto titulado "Anillo de funciones multisimétricas" identificado con el ID 00005024.

\section{Referencias}

[1] Aigner, M. (2007) A course in Enumeration. Graduate Texts in Mathematics, vol. 238, Springer Verlag, Berlin.

[2] Bergeron, F. (1990) "Combinatoire del polynômes orthogonaux classiques: Une approche unifiée”, European Journal of Combinatorics 11(5): 393 401.

[3] Bergeron, F.; Labelle, G.; Leroux, P. (1998) Combinatorial species and tree-like structures 67. Cambridge University Press, United Kingdom.

[4] Cartier, P.; Foata, D. (1969) Problèmes Combinatoires de Commutation et Réarrangements/Commutation and Rearrangements. Lecture Notes in Mathematics 85, Springer Verlag, Berlin.

[5] Díaz, R.; Pariguan, E. (2009) "Super, quantum and non-commutative species”, Afr. Diaspora J. Math. (N.S.) 8(1): 90-130.

[6] Joyal, A. (1981) "Une théorie combinatoire des séries formelles", Advances in Mathematics 42(1): 1-82.

[7] Lando, S.K. (2003) Lectures on Generating Functions. Student Mathematical Library, vol. 23, American Mathematical Society, Providence RI.

[8] Mac Lane, S. (1998) Categories for the Working Mathematician. Graduate Texts in Mathematics, vol. 5, Springer Verlag, Berlin.

[9] Mullin, R.; Rota, G.-C. (1970) "On the foundations of combinatorial theory III. Theory of binomial enumeration”, in: B. Harris (Ed.) Graph Theory and its Applications, Academic Press, New York: 167-213.

[10] Rota, G.-C. (1964) "On the foundations of combinatorial theory I. Theory of Möbius functions", Z. Wahrscheinlichkeitstheorie (Probability Theory and Related Fields) 2(4): 340-368. 
[11] Rota, G.-C.; Taylor, B.D. (1994) "The classical umbral calculus", SIAM Journal on Mathematical Analysis 25(2): 694-711.

[12] Stanley, R.P. (1999) "Enumerative combinatorics", Cambridge Studies in Advanced Mathematics 62(2): 51-100.

[13] Wehrhahn, K.H. (1992) Combinatorics: An Introduction. Carslaw Publications, Australia. 
